Fetal Diagnosis and Therapy

\title{
Clinical and Economic Impact of Adopting Noninvasive Prenatal Testing as a Primary Screening Method for Fetal Aneuploidies in the General Pregnancy Population
}

\author{
Emilia Kostenko $^{a}$ Frederic Chantraine $^{b}$ Katleen Vandeweyer ${ }^{c}$ \\ Maximilian Schmid ${ }^{d}$ Alex Lefevre $^{c}$ Deanna Hertz ${ }^{\mathrm{e}}$ Laura Zelle $^{\mathrm{e}}$ \\ Jose Luis Bartha ${ }^{f}$ Gian Carlo Di Renzo ${ }^{g}$ \\ ${ }^{a}$ Roche Sequencing Solutions, Inc., Prague, Czech Republic; ${ }^{b}$ Department of Obstetrics and Gynecology, CHR \\ Citadelle, Liège, Belgium; ' Roche Sequencing Solutions, Inc., Vilvoorde, Belgium; d Roche Sequencing Solutions, Inc.,

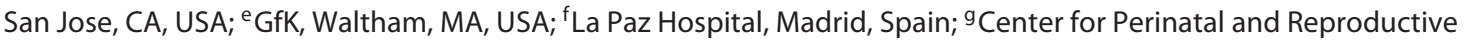 \\ Medicine, University of Perugia, Perugia, Italy
}

\section{Keywords}

Prenatal screening · Maternal serum screening •

Noninvasive prenatal diagnosis - Cost effectiveness .

Economic evaluation - Cell-free DNA · Genetic testing •

Fetal diagnosis · Down syndrome · Trisomy

\begin{abstract}
Objective: To evaluate the clinical and economic impact of adopting noninvasive prenatal testing (NIPT) using circulating cell-free DNA as a first-line screening method for trisomy 21,18 , and 13 in the general pregnancy population. Methods: A decision-analytical model was developed to assess the impact of adopting NIPT as a primary screening test compared to conventional screening methods. The model takes the Belgium perspective and includes only the direct medical cost of screening, diagnosis, and procedure-related complications. NIPT costs are EUR 260. Clinical outcomes and the cost per trisomy detected were assessed. Sensitivity analysis measured the impact of NIPT false-positive rate (FPR) on modelled results. Results: The cost per trisomy detected was EUR 63,016 for conventional screening versus EUR 66,633 for
\end{abstract}

\begin{tabular}{ll}
\hline KARGER & ( ) 2018 The Author(s) \\
& Published by S. Karger AG, Basel Oparger \\
E-Mail karger@karger.com & This article is licensed under the Creative Commons Attribution- \\
www.karger.com/fdt & NonCommercial-NoDerivatives 4.0 International License (CC BY- \\
NC-ND) (http://www.karger.com/Services/OpenAccessLicense). \\
Usage and distribution for commercial purposes as well as any dis- \\
tribution of modified material requires written permission.
\end{tabular}

NIPT, with a difference of EUR 3,617. NIPT reduced unnecessary invasive tests by $94.8 \%$, decreased procedure-related miscarriages by $90.8 \%$, and increased trisomies detected by $29.1 \%$. Increasing the FPR of NIPT (from $<0.01$ to $1.0 \%$ ) increased the average number of invasive procedures required to diagnose a trisomy from 2.2 to 4.5 , respectively. Conclusion: NIPT first-line screening at a reasonable cost is costeffective and provides better clinical outcomes. However, modelled results are dependent on the adoption of an NIPT with a low FPR.

(c) 2018 The Author(s)

Published by S. Karger AG, Basel

\section{Introduction}

Prenatal screening for trisomy 21 (Down syndrome, T21), trisomy 18 (Edwards syndrome, T18), and trisomy 13 (Patau syndrome, T13) is routinely offered to pregnant women in most European countries. The most frequently used approach is first-trimester screening (FTS), which combines nuchal translucency (NT) ultrasound measurement and maternal serum testing to assess the levels of 
human chorionic gonadotropin (hCG) and pregnancyassociated plasma protein $\mathrm{A}$ in combination with age and medical history $[1,2]$. The triple test (hCG, alpha-fetoprotein, and unconjugated estriol) is used to screen women presenting in the second trimester [3].

Conventional screening methods have significant limitations, resulting in missed identification of trisomies and a need for invasive testing which can lead to adverse events such as miscarriages. In Belgium, the observed overall performance of the conventional FTS program varies from $70 \%>90 \%$ sensitivity, with a $5 \%$ false-positive rate (FPR) [4].

In recent years, highly sensitive and specific noninvasive prenatal testing (NIPT) has become available. NIPT uses cell-free DNA from maternal plasma, which is a highly effective method of screening for Down syndrome and other autosomal trisomies [5]. There is strong clinical evidence supporting the use of NIPT in the general population [6-9], and major scientific societies have recently published guidelines supporting the use of NIPT as a first-line test in all pregnant women [10-12]. Health technology assessments and governmental bodies worldwide have found that NIPT is significantly more sensitive and specific than conventional screening methods and that providing NIPT screening to the general pregnancy population would be clinically superior to its second-line use following a positive conventional screening result [1316]. As a result, NIPT primary screening for all pregnant women was approved for reimbursement in Belgium starting on July 1, 2017 [16].

However, healthcare authorities in other countries still have concerns about the budget impact of implementing NIPT as a primary screening test. Economic analysis is needed to evaluate the financial impact of NIPT implementation in the public health system for the general pregnancy population. This study evaluates the potential clinical and economic consequences of adopting NIPT testing (Harmony ${ }^{\circledR}$ prenatal test, Roche) for T21, T18, and T13 in the general pregnancy population, using Belgium as a case study.

\section{Methods}

Using Microsoft Excel ${ }^{\circledR} 2010$ (Microsoft Corp., Redmond, WA, USA), we developed a decision-analytical model to compare various prenatal screening approaches for a hypothetical population of women with pregnancies reaching a gestational age of 10 weeks. Although NIPT has been validated in twin pregnancies [17], the model included only singleton pregnancies due to limited data to enable the inclusion of twin pregnancies. Women present- ing for aneuploidy screening were followed over the screening period, with costs of screening, diagnosis, and associated adverse events reported from the public health system perspective. Costs outside of the screening period (e.g., cost of trisomic births) were not included in the model.

The model consisted of two scenarios. Scenario 1 was conventional screening. All women who presented for screening received either FTS with NT ultrasound plus maternal serum screening of pregnancy-associated plasma protein A and hCG or second-trimester screening (STS) with maternal serum screening of alphafetoprotein, $\beta$-hCG, and unconjugated estriol, also known as the triple test. Second-trimester ultrasound was excluded from the model since it would occur independent of any previous screening tests and would not be eliminated from screening algorithms if NIPT was adopted. Scenario 2 was primary NIPT screening with NIPT as a first-line screening test. Patient flow diagrams for both scenarios are provided in Figure 1.

The model assumes a screening uptake rate of $78.87 \%$, with $78.10 \%$ of those receiving FTS only and $21.90 \%$ receiving STS only [4]. No women received both FTS and STS in the model. In realworld practice, there may exist a cohort of women receiving both FTS and STS, but published data are not available to quantify this cohort in Belgium.

In the conventional screening scenario, a small percentage (1\%) of pregnant women were assumed to go directly to invasive testing in place of screening either because it provides more certainty or based solely on age [4]. Women who presented for screening in the first trimester received FTS. Women presenting in the second trimester received STS with the triple test. The high-risk cutoff for conventional screening was assumed to be 1:300, a risk cutoff that is commonly used for FTS and was previously the practice in Belgium. Screening-positive women - those screening highrisk on either the FTS or STS - received an additional physician visit and were referred to invasive testing using either chorionic villus sampling or amniocentesis. A portion (12.5\%) of screeningpositive women indicated for invasive testing will opt to decline further screening. Women who receive invasive testing are at risk of procedure-related complications including miscarriage, leakage of amniotic fluid, and rare complications such as infection, prolonged rupture, feto-maternal hemorrhage, Rhesus alloimmunization, and fetal injury.

In the primary NIPT screening scenario, NIPT was used firstline as a replacement for FTS and STS for all pregnant women presenting for screening. Women who would have opted to go directly to invasive testing in the conventional screening scenario were assumed to receive NIPT instead. The result of NIPT indicates high or low probability of aneuploidy or, in some cases, "no result." NIPT "no result" outcomes are typically due to insufficient fetal fraction or high variance in cell-free DNA counts [6]. Women with a "no result" outcome - $3 \%$ of the NIPT tests in the model received repeat NIPT. Those with a second "no result" outcome, accounting for $37 \%$ of repeat NIPT, were referred to either conventional screening with serum markers or invasive testing. Women at high probability of aneuploidy after NIPT received an additional physician visit and were referred to invasive testing (chorionic villus sampling or amniocentesis), with a portion (12.5\%) opting to forego further screening. Women with a low probability of aneuploidy exited the model. Women who received invasive testing are at risk of procedure-related complications. 


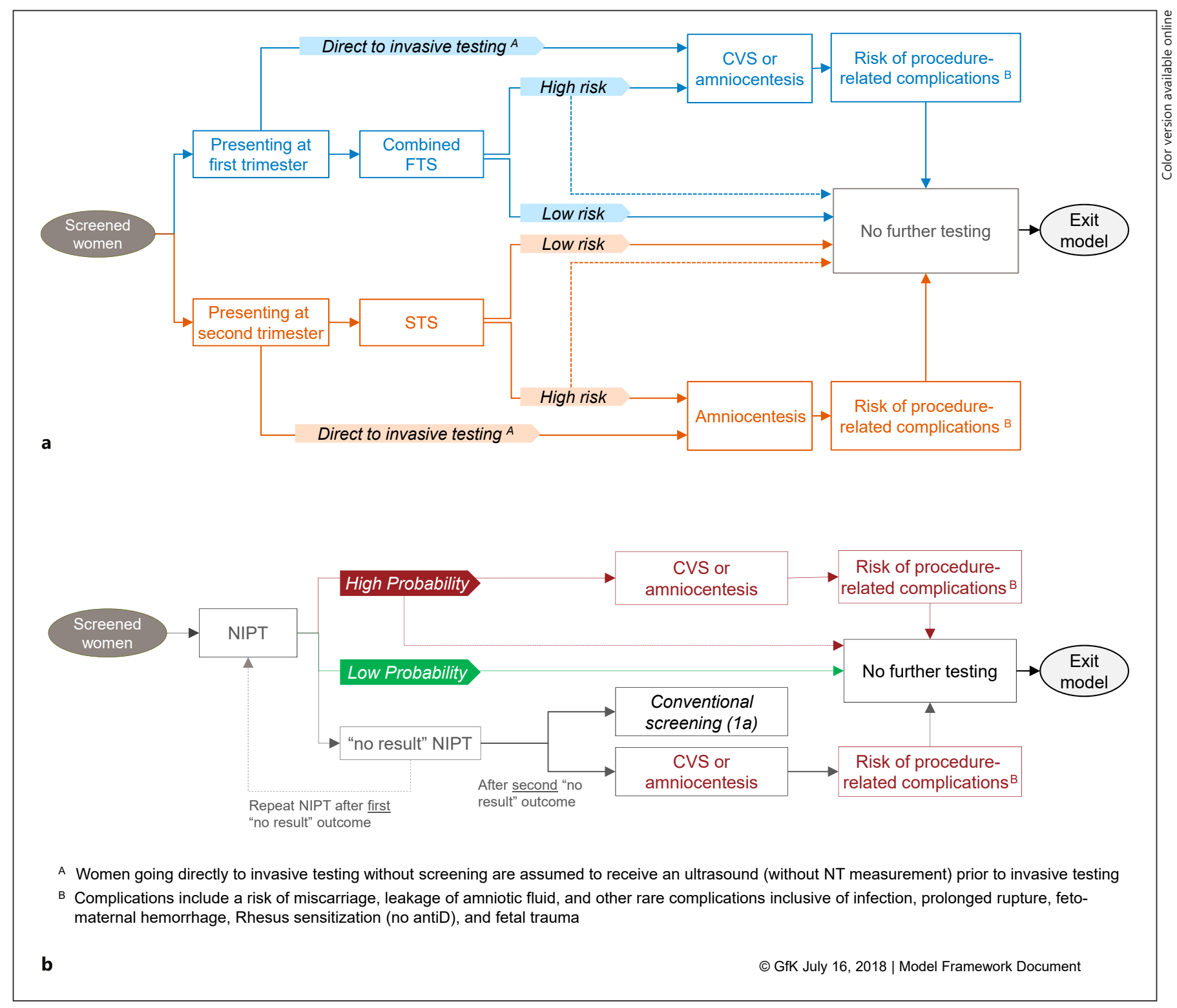

Fig. 1. Patient flow for conventional screening (a) and primary NIPT screening (b). CVS, chorionic villus sampling; FTS, first-trimester screening; NIPT, noninvasive prenatal testing; NT, nuchal translucency; STS, secondtrimester screening.

\section{Model Inputs}

Data to support population assumptions were derived from published sources and European Union (Eurostat) databases. Epidemiology, clinical practice, and test performance were sourced from the published literature. Aneuploidy incidence rates were calculated by taking the geometric mean, weighted by the population's age distribution [18]. Only the direct costs associated with screening, diagnosis, and associated adverse events were included in the model. Cost assumptions were primarily derived from the Belgian reimbursement tariffs [19] and the Belgium Health Care Knowledge Center NIPT health economic report [4]. Costs are expressed in 2018 EUR. Given the short-term focus of the analysis, costs were not discounted. Base case model inputs and uncertainty ranges can be found in Tables 1-3.

\section{Model Validation and Evaluating Uncertainty}

Four European experts in prenatal screening reviewed the model's clinical validity. The modelled results were compared to published trisomy screening models. The calculated number of invasive procedures was compared to the actual reported number of invasive procedures in Belgium. The background incidence of T21 calculated by the model was compared to estimated rates of T21 in an unscreened Belgium population. 
Table 1. Model inputs (epidemiology and patient flow probabilities)

\begin{tabular}{|c|c|c|}
\hline Variables & Base value & Range \\
\hline \multicolumn{3}{|l|}{ Epidemiology } \\
\hline Total number of pregnancies reaching 10 weeks GA & $131,567[4]$ & $105,254-157,880^{\mathrm{a}}$ \\
\hline Singleton pregnancies, $\%$ & $98.20[4]$ & $78.60-100.0 \%{ }^{\mathrm{a}}$ \\
\hline Incidence of T21, T18, and T13 - first trimester, $\%$ & $0.298,0.202,0.136[20]$ & $\pm 20 \%^{\mathrm{a}}$ \\
\hline Incidence of T21, T18, and T13 - second trimester, $\%$ & $0.202,0.045,0.013[20]$ & $\pm 20 \%^{\mathrm{a}}$ \\
\hline \multicolumn{3}{|l|}{ Screened women receiving the following tests by screening algorithm, \% } \\
\hline \multicolumn{3}{|l|}{ Conventional screening } \\
\hline FTS & $78.10[4]$ & $62.48-93.72^{\mathrm{a}}$ \\
\hline STS & $21.90[4]$ & $1-\mathrm{FTS}$ \\
\hline Invasive testing without prior screening & $1[4]$ & $0.70-1.04^{\mathrm{a}}$ \\
\hline Invasive testing following positive screen [4] & 87.50 & $70.00-100^{\mathrm{a}}$ \\
\hline Invasive testing following positive screening & $87.50[4]$ & $70.00-100^{\mathrm{a}}$ \\
\hline "No result" rate - 1st draw & $3.00[21]$ & $2.40-3.60^{\mathrm{a}}$ \\
\hline "No result" rate -2 nd draw & $37.00[21]$ & $29.60-44.40^{\mathrm{a}}$ \\
\hline Second "no result" to invasive testing (remainder to conventional screening) & $30.00^{\mathrm{b}}$ & $0-100$ \\
\hline Percentage of positive results receiving genetic counseling prior to invasive testing & $15.00^{\mathrm{b}}$ & $12.00-18.00^{\mathrm{a}}$ \\
\hline Procedure-related miscarriage & $0.50[22]$ & $0.50-1.00^{\mathrm{b}}$ \\
\hline Procedure-related leakage of amniotic fluid & $1.00[4]$ & $0.80-1.20^{\mathrm{a}}$ \\
\hline Additional rare complications of invasive testing & $1.00^{\mathrm{b}}$ & $0.80-1.20^{\mathrm{a}}$ \\
\hline
\end{tabular}

FTS, first-trimester screening; GA, gestational age; NIPT, noninvasive prenatal testing; STS, second-trimester screening. ${ }^{\text {a }}$ Sensitivity range of $\pm 20 \%$; does not exceed $100 \%$ nor the lower bound of $0 \%$. ${ }^{b}$ Model assumption based on expert opinion.

Table 2. Cost inputs

\begin{tabular}{lcc}
\hline Variables & Base value, EUR & Range \\
\hline Cost of FTS & $124.30[19]$ & $99.44-149.16^{\mathrm{a}}$ \\
Cost of STS & $43.76[19]$ & $35.01-52.51^{\mathrm{a}}$ \\
Cost of NIPT & $260.00[23]$ & - \\
Cost of CVS & $965.25[19]$ & $772.20-1,158.30^{\mathrm{a}}$ \\
Cost of amniocentesis & $965.25[19]$ & $772.20-1,158.30^{\mathrm{a}}$ \\
Cost of genetic counseling & $180.95[19]$ & $144.76-217.14^{\mathrm{a}}$ \\
Cost of treatment for procedure-related miscarriage & $107.64[19]$ & $86.11-129.17^{\mathrm{a}}$ \\
Cost of additional physician visit (high-risk results) & $25.43[19]$ & $20.34-30.52^{\mathrm{a}}$ \\
Cost of treatment for amniotic fluid leakage & $3,755.29[4]$ & $3,004.23-4,506.34^{\mathrm{a}}$ \\
Cost of treatment for rare complications & $1,213.98[4]^{\mathrm{b}}$ & $971.18-1,456.77^{\mathrm{a}}$ \\
\hline
\end{tabular}

CVS, chorionic villus sampling; FTS, first-trimester screening; NIPT, noninvasive prenatal testing; STS, second-trimester screening. ${ }^{a}$ Sensitivity range of $\pm 20 \%$; does not exceed $100 \%$ nor the lower bound of $0 \%$. ${ }^{b}$ Model assumption based on expert opinion and costs of 2-day hospital stay, laboratory testing, drugs, medical imaging, and supervision reported in the Belgium Health Care Knowledge Center report. 
Table 3. Test performance inputs

\begin{tabular}{|c|c|c|c|c|c|}
\hline Test performance inputs & Sensitivity, \% & Range, \% & Specificity, \% & Range, \% & Reference \\
\hline \multicolumn{6}{|l|}{ FTS } \\
\hline Trisomy 21 & 78.90 & $62.70-90.40$ & 94.60 & $94.20-94.90$ & {$[6]$} \\
\hline Trisomy 18 & 90.00 & $33.00-100$ & 94.00 & $92.00-100$ & {$[24]$} \\
\hline Trisomy 13 & 78.00 & $57.00-100$ & 92.00 & $92.00-98.00$ & {$[24]$} \\
\hline \multicolumn{6}{|l|}{ STS } \\
\hline Trisomy 21 & 80.50 & $60.00-100$ & 90.50 & $46.00-96.00$ & {$[24]$} \\
\hline Trisomy 18 & 63.50 & $53.00-86.00$ & 99.25 & $64.00-100$ & {$[24]$} \\
\hline Trisomy 13 & 50.00 & $40.00-60.00^{\mathrm{a}}$ & 64.00 & $51.20-76.80^{\mathrm{a}}$ & {$[24]$} \\
\hline \multicolumn{6}{|l|}{ NIPT } \\
\hline Trisomy 21 & 100 & $90.70-100$ & 99.90 & $99.90-100$ & {$[6]$} \\
\hline Trisomy 18 & 97.40 & $93.40-99.00$ & 99.98 & $99.95-99.99$ & {$[5]$} \\
\hline Trisomy 13 & 93.80 & $79.90-98.30$ & 99.98 & $99.94-99.99$ & {$[5]$} \\
\hline Invasive testing $^{\mathrm{c}}$ & $100^{\mathrm{b}}$ & $80.00-100^{\mathrm{a}}$ & $100^{\mathrm{b}}$ & $80.00-100^{\mathrm{a}}$ & \\
\hline
\end{tabular}

FTS, first-trimester screening; NIPT, noninvasive prenatal testing; STS, second-trimester screening. ${ }^{a}$ Sensitivity range of $\pm 20 \%$; does not exceed $100 \%$ nor the lower bound of $0 \%$. ${ }^{b}$ Model assumption based on expert opinion. ${ }^{c}$ Chorionic villus sampling and amniocentesis.

A one-way sensitivity analysis examined the impact of all model inputs on the resulting difference in cost per trisomy detected to account for the level of uncertainty associated with the input. Inputs were varied across their plausible ranges while holding other inputs constant at their base case value. The ranges used in the sensitivity analysis are based on the literature or by $\pm 20 \%$ in cases where data were not available (Tables 1-3).

While sensitivity is comparable among most NIPTs (>99\%), the FPR can differ significantly, ranging from $<0.1$ to $1.0 \%[5,25$, $26]$. To determine the impact of the variation in FPR on model results, a scenario analysis was performed. The FPR was adjusted based on reported ranges of NIPT, while all other inputs were held constant at the base case values.

\section{Results}

Based on an estimated annual pregnancy volume of 131,567 pregnancies reaching 10 weeks of gestation and a prenatal screening uptake rate of $78.87 \%, 101,899$ pregnant women were screened for T21, T18, and T13 in both scenarios of the model. Of these, 79,586 women presented in the first trimester and 22,313 in the second trimester.

In the conventional screening scenario, 1,019 (1\%) women received invasive testing without prior screening. Of the remaining women, 78,791 (77\%) received FTS and 22,090 (22\%) STS. A total of 8,788 (8.6\%) women screened either FTS- or STS-positive, with 7,690 (87.5\%) going on

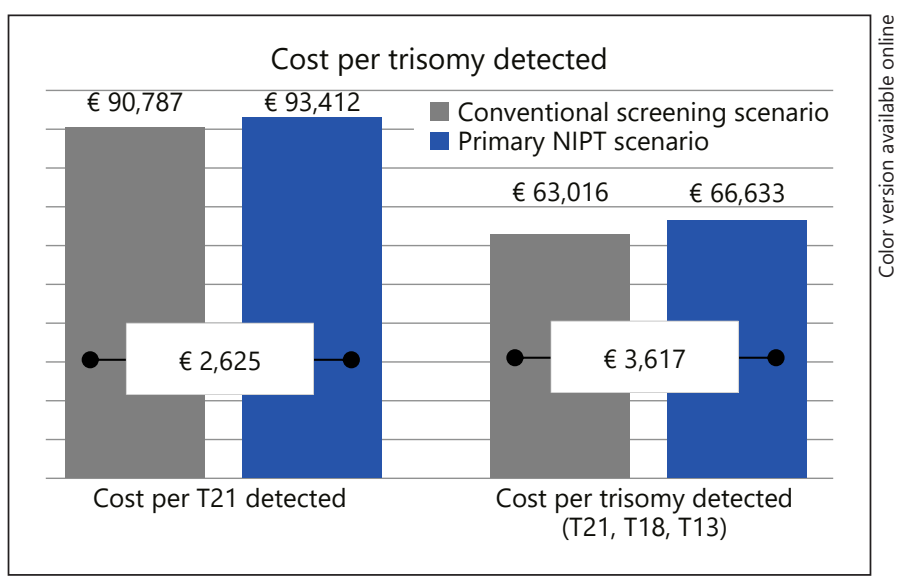

Fig. 2. Graph displaying cost-effectiveness, measured as the cost per trisomy detected, of the primary NIPT screening scenario versus the conventional screening scenario for both T21 detected and all trisomies detected (T21, T18, and T13). NIPT, noninvasive prenatal testing.

to receive invasive testing. In total, 8,709 women received an invasive test, with 8,426 (96.8\%) invasive tests indicating no trisomy 21,18 , or 13 pregnancies and thus being potentially avoidable. The average risk of procedurerelated miscarriages was $0.043 \%(44 / 101,899)$, and that of other procedure-related complications was $0.171 \%$ $(174 / 101,899)$ in the screened population. Conventional 
Table 4. Clinical outcomes

\begin{tabular}{llcr}
\hline Clinical outcomes & $\begin{array}{l}\text { Conventional } \\
\text { screening scenario }\end{array}$ & $\begin{array}{l}\text { Primary NIPT } \\
\text { screening scenario }\end{array}$ & $\begin{array}{l}\text { Difference } \\
\text { (\% difference) }\end{array}$ \\
\hline Number of invasive tests & 8,709 & 797 & $-7,912(-90.8)$ \\
Number of unnecessary invasive tests & 8,426 & 437 & $-7,989(-94.8)$ \\
Procedure-related miscarriages & 44 & 4 & $-40(-90.8)$ \\
Procedure-related leakage of amniotic fluid & 87 & 8 & $-79(-90.8)$ \\
Procedure-related rare complications & 87 & 8 & $-79(-90.8)$ \\
Missed trisomies & 77 & 5 & $-72(-93.6)$ \\
Trisomy 21 detected (detection rate, \%) & $221(79)$ & $293(100)$ & $72(32.6)$ \\
Trisomy 18 detected (detection rate, \%) & $74(87)$ & $87(97)$ & $13(17.8)$ \\
Trisomy 13 detected (detection rate, \%) & $24(75)$ & $31(94)$ & $7(30.6)$ \\
Total trisomies detected (detection rate, \%) & $318(81)$ & $411(99)$ & $93(29.1)$ \\
\hline
\end{tabular}

NIPT, noninvasive prenatal testing.

Table 5. Impact of false-positive rate on cost, invasive procedures, and procedure-related miscarriages

\begin{tabular}{lllll}
\hline & \multicolumn{4}{l}{ False-positive rate (base case) } \\
\cline { 2 - 5 } & $0.1 \%$ & $0.3 \%$ & $0.6 \%$ & $1 \%$ \\
\hline Incremental cost per trisomy detected, EUR & 3,617 & 4,199 & 4,889 & 5,808 \\
Invasive tests required to diagnose one trisomy & 2.2 & 2.8 & 3.6 & 4.5 \\
Procedure-related miscarriages avoided & 40 & 38 & 37 & 35
\end{tabular}

screening detected $81 \%$ of trisomies 21,18 , and 13 , missing 77 trisomies. On average, 30.8 invasive tests were required to detect one trisomy at a cost of EUR 63,016 per all three trisomies (T21, T18, T13) detected and EUR 90,787 per T21 detected (Fig. 2). Clinical and cost-effectiveness outcomes are presented in Table 4 and Figure 2, respectively.

In the primary NIPT screening scenario, all screened women received an NIPT test, 79,586 in the first trimester and 22,313 in the second trimester. Of the women receiving a second NIPT "no result," 792 women received either a FTS or STS follow-up screen and 339 women received an invasive test. A total of $523(0.5 \%)$ women screened positive, with $458(87.5 \%)$ going on to receive invasive testing. In total, 797 women received invasive testing, with 437 (54.9\%) invasive tests being avoidable due to a false-positive or no result. The average risk of procedurerelated miscarriages was $0.0039 \%(4 / 101,899)$, and that of other procedure-related complications was $0.0157 \%$ $(16 / 101,899)$ in the screened population. The reduction in procedure-related risk was a direct result of less invasive procedures being performed in the NIPT primary screening scenario and not related to the risk per proce- dure, which was assumed to be the same between scenarios. NIPT primary screening detected $99 \%$ of trisomies 21,18 , and 13, missing 5 trisomies. On average, 2.2 invasive tests were required to detect one trisomy at a cost of EUR 66,633 per all three trisomies detected and EUR 93,412 per T21 detected.

Compared to the conventional screening scenario, the primary NIPT screening scenario demonstrated efficient and more effective screening, reducing the number of invasive tests required to detect a trisomy by $92.8 \%$, from 30.8 to 2.2 , while improving the overall detection rate (from 81 to 99\%) and reducing missed trisomies (from 77 to $5 \%$ ). As a result, NIPT reduced the risk of procedurerelated complications by $90.8 \%$, avoiding an estimated 40 procedure-related miscarriages annually. At a cost of EUR 260 for the NIPT test, the difference in cost per trisomy diagnosed was estimated to be EUR 3,617 per trisomy diagnosed (Fig. 2).

\section{Validation and Uncertainty Analysis Results}

The model's predicted volume of invasive tests for the conventional screening scenario of 8,709 aligns with the 7,586 invasive tests reported in Belgium for 2011 [4]. Ad- 


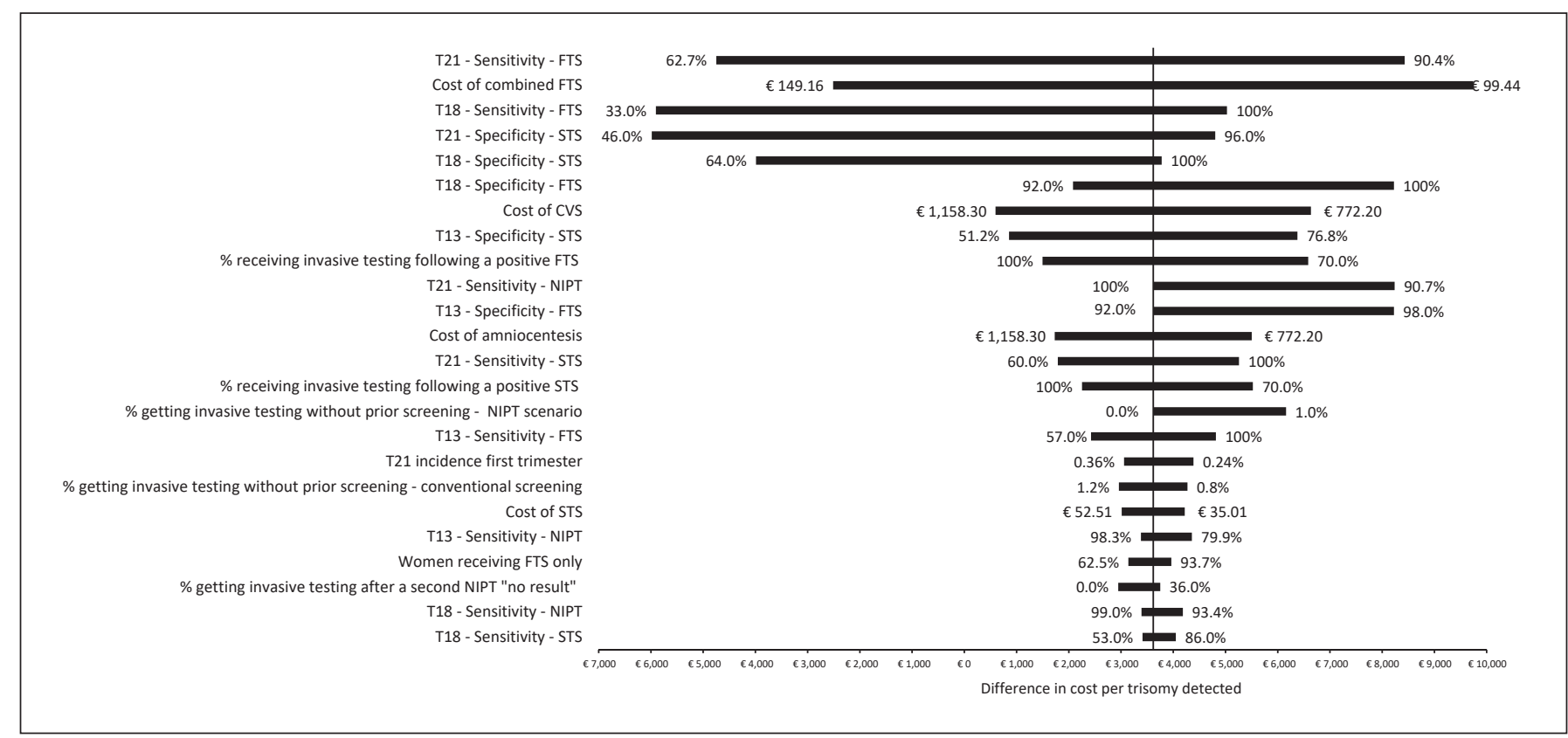

Fig. 3. Graph displaying the outcomes of the one-way sensitivity analysis, with a ranking of model inputs having the greatest impact on model results. Model inputs are along the vertical axis. The model outcome, difference in cost per trisomy detected between conventional screening and NIPT, is displayed on the horizontal axis. The

ditionally, the model's incidence of T21 is 293 , which compares favorably with the estimated 224 T21 births without screening in Belgium, based on 2012 figures [4]. The modelled positive predictive value (PPV) for the conventional screening scenario was $3.2 \%^{1}$ compared to $78.6 \%{ }^{2}$ for the NIPT primary screening scenario. This is comparable to the PPVs of $3.4 \%$ for standard screening and $80.9 \%$ for NIPT screening reported by Norton et al. [6].

Uncertainty in the test performance of conventional FTS and STS had the greatest impact on the difference in cost per trisomy detected. An extreme case analysis was conducted assuming the best and worst possible screening performances for the conventional screening scenario. The difference in cost per trisomy detected ranged from cost savings of EUR 51,723 for NIPT compared to conventional screening at the lowest plausible test performance to an added cost of EUR 23,911 for NIPT com-

1 The PPV of the conventional screening algorithm was calculated based on 318 detected trisomies divided by 8,788 women screened positive plus 1,109 women going directly to invasive testing.

2 The PPV of the NIPT primary screening algorithm was calculated based on 411 detected trisomies divided by 523 women screened positive.

NIPT as a Primary Screening Method for Fetal Aneuploidies bar labels show the range in model inputs, while the bar displays the associated range in outcome. Thus, wider bars indicate that the model input range has a greater impact on model results. CVS, chorionic villus sampling; FTS, first-trimester screening; NIPT, noninvasive prenatal testing; STS, second-trimester screening.

pared to conventional screening at the highest plausible test performance across both FTS and STS for all trisomies. The tornado plot (Fig. 3) provides the results of the most influential inputs, their associated uncertainty range, and the outcome for the difference in cost per trisomy detected.

\section{Scenario Analysis}

A scenario analysis was performed to determine the impact of different FPRs on the cost and clinical outcomes in the model (Table 5). The analysis showed that, within the plausible range of FPRs, the number of invasive tests required for every diagnosed trisomy increased from 2.2 in the base case to 3.7 at the highest FPR. This resulted in an increase in cost, from a base cost of EUR 3,617 to EUR 5,808 at the highest plausible FPR, as well as an increase in the incidence of procedure-related complications.

For the base case, the model assumed that the uptake of invasive testing following a positive screen was the same for all screening modalities. However, due to the improved test performance of NIPT, more women may choose to proceed to invasive testing following a positive NIPT result. A scenario analysis was performed to look at 
the relationship between the uptake of invasive testing following a positive NIPT and the cost per trisomy detected. The analysis showed a positive linear relationship with a slope of 9.58. Thus, for every percent increase in women proceeding to invasive testing following a positive NIPT result, the incremental cost per trisomy detected increased by EUR 9.58.

Additionally, the model assumed that women previously proceeding to invasive testing without a prior screen $-1 \%$ of the screened population in the FTS scenario - will instead opt for NIPT due to improved test performance and added certainty. However, there are clinical situations where NIPT may not be chosen or even offered and women will go directly to invasive testing. Assuming a similar percentage $(1 \%)$ of women receiving invasive testing prior to screening in the NIPT scenario, the resulting cost per trisomy detected was EUR 66,633 for an incremental cost per trisomy detected of EUR 6,160 (Fig. 3).

\section{Discussion}

There is an unmet need for a more accurate screening approach due to missed trisomies, high numbers of avoidable invasive tests, and resulting complications associated with conventional screening. By means of a case study of Belgium, which recently approved and funded NIPT as a first-line screening protocol, this analysis demonstrated that introducing NIPT for the general population is a cost-effective screening approach for public health systems. At a cost of EUR 260 per NIPT, effectiveness gains and reductions in adverse events come at a reasonable increase in cost. When NIPT is used as first-line screening in all pregnant women, the clinical benefits include very few unnecessary invasive tests, reduced false-positive results, more trisomies detected as early as at 10 weeks of gestation, and fewer missed trisomy diagnoses.

The results of our study also highlight the need to consider the variability in FPRs reported for different NIPTs. The FPR has a substantial impact on clinical management and, consequently, on costs. NIPTs with higher FPRs reduce efficiency, requiring more invasive tests to detect one trisomy. Higher invasive procedures result in higher costs as well as adverse events such as procedure-related miscarriages. Therefore, selection of the NIPT with the lowest FPR will ensure the cost-effectiveness of this screening approach.

One other study has estimated the impact of NIPT primary screening in Belgium. A 2014 analysis reported by Neyt et al. [1] and Hulstaert et al. [4] estimated the cost consequence of introducing NIPT for the detection of T21 in Belgium. The analysis concluded that the appropriate use of NIPT clearly improved the risk-benefit ratio of prenatal screening and that first-line use was clinically preferable to second-line use, but that it increased the cost per T21 detected. The study estimated a conventional screening cost and NIPT primary screening cost of EUR 86,944 and EUR 236,436 per T21, respectively.

The conventional screening cost was similar in this analysis, which, if limited to T21, was EUR 90,787 per T21 detected. The difference in the cost of NIPT primary screening between the two studies is primarily due the cost of NIPT, which was assumed to be EUR 460 per test in the Neyt et al. [1] study, but is now known to be EUR 260. There are also structural differences between the two models, such as the inclusion of trisomies 18 and 13 and the lower performance of STS, resulting in a higher FPR for conventional screening. Additionally, Neyt et al. [1] assumed the cost of first-trimester ultrasound with NT measurement within the NIPT screening scenario while the current model assumed that NIPT will replace FTS for aneuploidy screening, thus excluding the cost of the NT ultrasound.

The first-trimester ultrasound is performed for a wide range of purposes, including but not limited to NT measure, fetal viability, dating of pregnancy, and to detect major malformations (e.g., acrania or megacystis) and the number of fetuses, and will continue in clinical practice regardless of aneuploidy screening method. The analysis assumed that for trisomy screening with first-line NIPT, NT measurement would not be needed [27]. While firsttrimester ultrasound will be performed for other reasons, its costs will not be part of trisomy screening. A scenario analysis was run to assess the cost of adding the first-trimester ultrasound without the NT to the primary NIPT scenario and resulted in a cost of EUR 75,482 per all three trisomies detected and a cost of EUR 105,817 for T21 detected. However, there is no additional clinical benefit for detection of trisomies when the ultrasound is included.

Several other studies have estimated the economic impact of NIPT first-line testing in prenatal screening programs. In the Netherlands, Beulen et al. [28] developed an economic model to compare the costs and outcomes of current clinical practice using conventional screening compared with NIPT first-line and second-line screening. At a cost of EUR 254 or less, NIPT first-line screening became the dominant approach, resulting in lower cost and improved clinical outcomes. In the US, Benn et al.'s [11] analysis of NIPT as a first-line test in the general population showed increased T21, T18, and T13 detection 
and can be economically justified at a break-even cost of USD 744. Fairbrother et al. [29] conducted a cost-effectiveness analysis of NIPT in the general population versus FTS, assuming a societal perspective inclusive of both direct and indirect costs and inclusive of T21, T18, and T13. The study found that at a cost below USD 453 there was cost saving and that a cost of USD 665 provided the same cost per trisomy detected as FTS. Similarly, an analysis by Walker et al. [30] reported a societal perspective breakeven cost of USD 619, with first-line NIPT for all women the dominant approach. These analyses support the findings from the current study showing improved clinical performance at a reasonable cost per trisomy detected.

Despite the evidence supporting NIPT first-line screening as cost-effective, other published studies have found that the improved clinical benefits of NIPT first-line screening are too costly $[31,32]$. The assumed cost of NIPT has a significant impact on cost-effectiveness analyses. The cost of NIPT tended to be higher in prior published studies than reported in this study. The actual cost of NIPT has decreased significantly since its launch and is more in line with the reported cost in the current study, which represents the actual cost to Belgium's National Health Service. Additionally, published studies differ in the screening algorithms being compared as well as in the inclusion of T18 and T13, which are rarely considered.

This analysis uses Belgium's National Health Service as a case study due to their decision to cover NIPT as a primary screening modality and the availability of detailed data that informed that decision and the inputs for this model. However, we realize that clinical practice can vary greatly across countries, which makes the results of this analysis difficult to generalize. We attempted to address some of these variations in order to allow more generalizability of the study results. In Belgium, the uptake of prenatal screening is nearly $80 \%$. However, the uptake of screening across European countries ranges from as low as $30 \%$ in the Netherlands to $95 \%$ in Denmark [33]. While variation in screening uptake will impact the number of trisomies detected and the number of procedure-related complications, it does not impact the primary model outcomes, i.e., cost per trisomy detected or detection rate. Due to a lack of quality control, Belgium has wide variation in the performance of NT and FTS. However, this analysis does not use Belgium-specific test performance, but instead relies on a large randomized controlled trial and meta-analysis to inform test performance across all screening modalities. Additionally, the sensitivity analysis performed an extreme case analysis, given the wide range of likely performance reported in the literature. Ad-

NIPT as a Primary Screening Method for Fetal Aneuploidies ditionally in Belgium, approximately $22 \%$ of pregnant women who presented during the second trimester and prior to the approval of NIPT received STS, which tends to have poorer test performance than FTS. However, in other countries STS is used primarily as a residual test, with most women presenting in the first trimester. Assuming that all women receive FTS, the model resulted in an increase in the cost per trisomy detected of EUR 4,074 versus baseline results of EUR 3,617.

A key limitation of this analysis is the conservative timeframe, limiting the analysis to the short-term cost of screening and diagnosis, which allows for greater predictability of results, but does not account for the costs over the lifetime of a child born with a trisomy. However, screening programs aim to facilitate informed reproductive choice rather than to prevent costs associated with trisomy live births. Therefore, we decided to base this analysis on the cost of screening and diagnosis rather than the long-term decisions and associated costs. Other studies have shown that the inclusion of long-term costs would be expected to improve the cost-effectiveness of NIPT $[29,30]$. Another limitation is the uncertainty of test performance for conventional screening for T18 and T13. There is wide variation in the literature given the rare occurrence of these conditions. The uncertainty is addressed by using the best available data from a metaanalysis and conducting a sensitivity analysis based on the best- and worst-case scenarios for testing performance. Due to a lack of data, T18 and T13 are frequently excluded from published models. Therefore, we added the cost per T21 detection for comparative purposes. However, this analysis does not accurately reflect a NIPT testing offering T21 only as both the cost and resources used may differ from the current analysis. The study is further limited by virtue of being a hypothetical model with a theoretical cohort of pregnant women. Assumptions made within the model may not perfectly align with actual realworld circumstances.

\section{Conclusion}

NIPT at the appropriate cost is a cost-effective firstline screening strategy for common fetal trisomies in the general pregnancy population. As compared to conventional screening, NIPT primary screening increases overall trisomy detection rates and provides more efficient referral to invasive testing, leading to a reduction in the number of procedure-related miscarriages and other procedure-related complications, at a similar cost per triso- 
my detected. Furthermore, NIPT with a low FPR is essential to achieving the clinical benefits and cost-effectiveness outcomes predicted by this analysis.

\section{Statement of Ethics}

Ethics committee approval and informed consent were not considered to be necessary for this study as it did not involve human subjects: the cohort was hypothetical.

\section{Disclosure Statement}

Roche Sequencing Solutions, Inc., manufacturer of the Harmony prenatal test, funded this research. E. Kostenko, K. Vandeweyer, M. Schmid, and A. Lefevre are employees of Roche Sequencing Solutions, Inc. D. Hertz and L. Zelle are employees of GfK, the consultancy that received a fee for this research. F. Chantraine, J.L. Bartha, and G.C. Di Renzo have previously received consulting fees from Roche, but no compensation was received for this research.

\section{References}

1 Neyt M, Hulstaert F, Gyselaers W: Introducing the non-invasive prenatal test for trisomy 21 in Belgium: a cost-consequences analysis. BMJ Open 2014;4:e005922.

2 European Surveillance of Congenital Anomalies (EUROCAT): EUROCAT Special Report: Prenatal Screening Policies in Europe 2010. EUROCAT Central Registry, University of Ulster, 2010.

3 Verloes A, Gillerot Y, van Maldergem L, Schoos R, Herens C, Jamar M, Dideberg V, Lesenfants S, Koulischer L: Major decrease in the incidence of trisomy 21 at birth in south Belgium: mass impact of triple test? Eur J Hum Genet 2001;9:1-4.

4 Hulstaert F, Neyt M, Gyselaers W: The noninvasive prenatal test (NIPT) for trisomy 21 - health economic aspects. Health Technology Assessment (HTA). Brussels, Belgian Health Care Knowledge Centre (KCE), 2014.

5 Stokowski R, Wang E, White K, Batey A, Jacobsson B, Brar H, Balanarasimha M, Hollemon D, Sparks A, Nicolaides K, Musci TJ: Clinical performance of non-invasive prenatal testing (NIPT) using targeted cell-free DNA analysis in maternal plasma with microarrays or next generation sequencing (NGS) is consistent across multiple controlled clinical studies. Prenat Diagn 2015;35:1243-1246.

6 Norton ME, Jacobsson B, Swamy GK, Laurent LC, Ranzini AC, Brar H, Tomlinson MW, Pereira L, Spitz JL, Hollemon D, Cuckle H: Cell-free DNA analysis for noninvasive examination of trisomy. N Engl J Med 2015;372: 1589-1597.

7 Nicolaides KH, Syngelaki A, Ashoor G, Birdir C, Touzet G: Noninvasive prenatal testing for fetal trisomies in a routinely screened firsttrimester population. Am J Obstet Gynecol 2012;207:374.e1-e6.

8 Gil MM, Quezada MS, Bregant B, Ferraro M, Nicolaides KH: Implementation of maternal blood cell-free DNA testing in early screening for aneuploidies. Ultrasound Obstet Gynecol 2013;42:34-40.
9 Verweij EJ, Jacobsson B, Scheltema PA, Boer MA, Hoffer MJ, Hollemon D, Westgren M, Song K, Oepkes D: European Non-Invasive Trisomy Evaluation (EU-NITE) study: a multicenter prospective cohort study for non-invasive fetal trisomy 21 testing. Prenat Diagn 2013;33:996-1001.

10 American College of Obstetricians and Gynecologists: Committee Opinion No. 640: Cellfree DNA screening for fetal aneuploidy. Obstet Gynecol 2015;126:e31-e37.

11 Benn P, Borrell A, Chiu RW, Cuckle H, Dugoff L, Faas B, Gross S, Huang T, Johnson J, Maymon R, Norton M: Position statement from the Chromosome Abnormality Screening Committee on behalf of the Board of the International Society for Prenatal Diagnosis. Prenat Diagn 2015;35:725-734.

12 Gregg AR, Skotko BG, Benkendorf JL, Monaghan KG, Bajaj K, Best RG, Klugman S, Watson MS: Noninvasive prenatal screening for fetal aneuploidy, 2016 update: a position statement of the American College of Medical Genetics and Genomics. Genet Med 2016;18: 1056-1065.

13 Publication of the Superior Health Council No. 8912 (HGR-CSS, report. 2014): Implementation of non-invasive prenatal genetic screening for trisomy 21 (Down Syndrome) in the practice of health care in Belgium. May 7, 2014.

14 Health Council of the Netherlands: Prenatal screening. The Hague, Health Council, 2016, publication No. 2016/19, ISBN 978-94-6281088-4.

15 Italian Ministry of Health: Linee guida: screening prenatale non invasive basato sul DNA [guidelines: DNA based noninvasive prenatal testing]. July 3, 2015.

16 Belgium Monitor 25.10.2017. http://www. vbs-gbs.org/fileadmin/user_upload/ e-specialist/2017/KB-AR_2017.10.15_-_BSMB_2017.10.25_NIPT.pdf.

17 Gil MD, Quezada MS, Bregant B, Syngelaki A, Nicolaides KH: Cell-free DNA analysis for trisomy risk assessment in first-trimester twin pregnancies. Fetal Diagn Ther 2014;35:204211.
18 Cuckle HS, Wald NJ, Thompson SG: Estimating a woman's risk of having a pregnancy associated with Down's syndrome using her age and serum alpha-fetoprotein level. Br J Obstet Gynaecol 1987;94:387-402.

19 NomenSoft (Belgium): National Institute for Health and Disability Insurance (INAMI). http://www.riziv.fgov.be/nl/themas/kost-terugbetaling/door-ziekenfonds/individueleverzorging/honoraires/Paginas/default. aspx\#.W15vW6inHIV F (accessed July 2016).

20 Snijders RJ, Holzgreve W, Cuckle H, Nicolaides KH: Maternal age-specific risks for trisomies at 9-14 weeks' gestation. Prenat Diagn 1994;14:543-552.

21 Gil MM, Revello R, Poon LC, Akolekar R, Nicolaides KH: Clinical implementation of routine screening for fetal trisomies in the UK NHS: cell-free DNA test contingent on results from first-trimester combined test. Ultrasound Obstet Gynecol 2016;47:45-52.

22 Tabor A, Alfirevic Z: Update on procedurerelated risks for prenatal diagnosis techniques. Fetal Diagn Ther 2010;27:1-7.

23 Institut national d'assurance maladie invalidité (INAMI): CSS 2017/175. May 29, 2017.

24 Institute of Health Economics: First and Second Trimester Prenatal Screening for Trisomies 13, 18, and 21 and Open Neural Tube Defects. Edmonton, Institute of Health Economics, 2012.

25 Palomaki GE, Kloza EM, Lambert-Messerlian GM, Haddow JE, Neveux LM, Ehrich M, van den Boom D, Bombard AT, Deciu C, Grody WW, Nelson SF: DNA sequencing of maternal plasma to detect Down syndrome: an international clinical validation study. Genet Med 2011;13:913-920.

26 Taylor-Phillips S, Freeman K, Geppert J, Agbebiyi A, Uthman OA, Madan J, Clarke A, Quenby S, Clarke A: Accuracy of non-invasive prenatal testing using cell-free DNA for detection of Down, Edwards and Patau syndromes: a systematic review and meta-analysis. BMJ Open 2016;6:e010002. 
27 Langlois S, Johnson J, Audibert F, Gekas J, Forest JC, Caron A, Harrington K, Pastuck M, Meddour H, Tétu A, Little J: Comparison of first tier cell-free DNA screening for common aneuploidies with conventional publically funded screening. Prenat Diagn 2017;37: 1238-1244.

28 Beulen L, Grutters JP, Faas BH, Feenstra I, van Vugt JM, Bekker MN: The consequences of implementing non-invasive prenatal testing in Dutch national health care: a cost-effectiveness analysis. Eur J Obstet Reprod Biol 2014; 182:53-61.
29 Fairbrother G, Burigo J, Sharon T, Song K: Prenatal screening for fetal aneuploidies with cell-free DNA in the general pregnancy population: a cost-effectiveness analysis. J Matern Fetal Neonatal Med 2016;29:1160-1164.

30 Walker BS, Jackson BR, LaGrave D, Ashwood ER, Schmidt RL: A cost-effectiveness analysis of cell free DNA as a replacement for serum screening for Down syndrome. Prenat Diagn 2015;35:440-446.

31 Morris S, Karlsen S, Chung N, Hill M, Chitty LS: Model-based analysis of costs and outcomes of non-invasive prenatal testing for Down's syndrome using cell free fetal DNA in the UK National Health Service. PLoS One 2014;9:e93559.
32 Nshimyumukiza L, Beaumont JA, Duplantie J, Langlois S, Little J, Audibert F, McCabe C, Gekas J, Giguère Y, Gagné C, Reinharz D, Rousseau F: Cell-free DNA-based non-invasive prenatal screening for common aneuploidies in a Canadian province: a cost-effectiveness analysis. J Obstet Gynaecol Can 2018; 40:48-60.

33 Crombag NM, Bensing JM, Iedema-Kuiper R, Schielen PC, Visser GH: Determinants affecting pregnant women's utilization of prenatal screening for Down syndrome: a review of the literature. J Matern Fetal Neonatal Med 2013; 26:1676-1681. 